Accepted author's manuscript. Published in final edited form as: Journal of Sleep Research 2020 (in press); e13169. Publisher DOI: 10.1111/jsr.13169

\title{
1 Treating insomnia in Swiss primary care practices: a survey study 2 based on case vignettes
}

3
Stefanie Linder ${ }^{1}$, Simone B. Duss ${ }^{2}$, Charles Dvoř́k ${ }^{3}$, Christoph Merlo ${ }^{3,4}$, Stefan Essig ${ }^{3,4}$, Kali Tal $^{1}$, Cinzia Del Giovane $^{1}$, Lamprini Syrogiannouli ${ }^{1}$, Raphael Heinzer ${ }^{5}$, Christoph Nissen ${ }^{6}$, Claudio L. A. Bassetti ${ }^{2}$, Reto Auer ${ }^{1,7}$, Micheline Maire ${ }^{1}$

${ }^{1}$ Institute of Primary Health Care (BIHAM), University of Bern, Switzerland.

${ }^{2}$ Sleep-Wake-Epilepsy Center, Department of Neurology, University Hospital (Inselspital) and University of Bern, Bern, Switzerland.

${ }^{3}$ Sentinella - Swiss Epidemiological System - Federal Office of Public Health FOPH, Bern, Switzerland.

${ }^{4}$ Institute of Primary and Community Care, Lucerne, Switzerland.

${ }^{5}$ Center for Investigation and Research in Sleep, Pulmonary Department, University Hospital of Lausanne, Lausanne, Switzerland.

${ }^{6}$ University Hospital of Psychiatry and Psychotherapy, Bern, Switzerland.

${ }^{7}$ Center for Primary Care and Public Health (Unisanté), University of Lausanne, Lausanne, Switzerland.

Correspondence:

Micheline Maire, $\mathrm{PhD}$

Institute of primary health care (BIHAM), University of Bern

Mittelstrasse 43

CH - 3012 Bern

micheline.maire@biham.unibe.ch

+41316315866

\section{Number of words:}

- Number of references: 32

- Word count text body: 3357 words 
1 Conflict of interest: SL, MM, RA report no conflict of interest. CN has received speaker honoraria 2 from Vanda Pharmaceuticals and has served on an advisory board of Lundbeck. RH is a member of 3 the medical advisory board of Dreem and NightBalance-Philips, received a grant from the Ligue

4 pulmonaire vaudoise, and speaker's fees from Nightbalance-Philips.

5 Author contribution: MM, RA and SL have contributed to the study concept, developed the survey, 6 and acquired, analyzed and interpreted data; they also drafted and revised the manuscript. CN, SD, RH 7 and CB helped develop the case vignettes and revised the manuscript. CDG and LS analyzed and 8 interpreted data. CD, CM and SE helped revise the survey and the manuscript. KT helped draft and 9 revise the manuscript. 


\section{Summary}

2 Guidelines recommend cognitive behavioral therapy for insomnia (CBT-I) as first-line treatment for

3 chronic insomnia, but it is not clear how many primary care physicians (PCPs) in Switzerland

4 prescribe this treatment. We created a survey that asked PCPs how they would treat chronic insomnia

5 and how much they knew about CBT-I. The survey included two case vignettes that described patients

6 with chronic insomnia, one with and one without comorbid depression. PCPs also answered general

7 questions about treating chronic insomnia and about CBT-I and CBT-I providers. Of the 820 Swiss

8 PCPs we invited, 395 (48\%) completed the survey (mean age 54; 70\% male); 87\% of PCPs prescribed

9 sleep hygiene and $65 \%$ phytopharmaceuticals for the patient who had only chronic insomnia; $95 \%$

10 prescribed antidepressants for the patient who had comorbid depression. In each case, $20 \%$ of PCPs

11 prescribed benzodiazepines or benzodiazepine receptor agonists, $8 \%$ prescribed CBT-I, $68 \%$ said they

12 knew little about CBT-I, and 78\% did not know a CBT-I provider. In the clinical case vignettes, most

13 PCPs treated chronic insomnia with phytopharmaceuticals and sleep hygiene despite their lack of

14 efficacy, but PCPs rarely prescribed CBT-I, felt they knew little about it, and usually knew no CBT-I

15 providers. PCPs need more information about the benefits of CBT-I and local CBT-I providers and

16 dedicated initiatives to implement CBT-I in order to reduce the number of patients who are prescribed

17 ineffective and potentially harmful medications.

18 Keywords: chronic insomnia, treatment, primary care, cognitive behavioral therapy for chronic

19 insomnia 


\section{Introduction}

2 Chronic insomnia is common in industrialized countries; prevalence averages about $10 \%$, though the number of affected people ranges from 6\% to 19\% in European countries (Calem et al., 2012; Leger,

4 Guilleminault, Dreyfus, Delahaye, \& Paillard, 2000; Marschall, Hildebrandt, Sydow, \& Nolting, 2017). DSM-5 defines chronic insomnia as subjective sleep disturbance at least 3 nights/week for at least 3 months, with concomitant daytime impairment (Association, 2013). Chronic insomnia lowers patient quality of life and poses a significant burden on the health care system (Delini-Stula, Bischof, \& Holsboer-Trachsler, 2007; Novak, Mucsi, Shapiro, Rethelyi, \& Kopp, 2004). It is also associated with medical and psychiatric conditions like cardiovascular disease and depression (Baglioni, Spiegelhalder, Nissen, \& Riemann, 2011; Li, Zhang, Hou, \& Tang, 2014). Chronic insomnia and depression are closely linked: depressed patients usually have altered sleep and chronic insomnia patients have twice the risk of developing depression (Baglioni et al., 2011; Riemann, Krone, Wulff, \& Nissen, 2020). The conditions share characteristics like hyperarousal, but each can arise and persist independently (Baglioni et al., 2011). Researchers once considered insomnia a symptom or consequence of depression, but more recent studies suggest it be treated separately (Baglioni et al., 2011). The DSM-5 now defines chronic insomnia independently from associated conditions and no longer distinguishes primary and secondary insomnia (Association, 2013). However, primary care physicians' (PCPs) practice has been slower to change (Sivertsen, Nordhus, Bjorvatn, \& Pallesen, 2010).

European Sleep Research Society's (ESRS) guidelines for treating chronic insomnia recommend first-line treatment with cognitive behavioral therapy for insomnia (CBT-I) (Riemann et al., 2017), which usually combines psychoeducation/sleep hygiene, relaxation training, stimulus control, sleep restriction, and cognitive therapy (Riemann \& Perlis, 2009). Sleep restriction has been found effective also when used alone (Krieger et al., 2019; Miller et al., 2014). If patients cannot access CBT-I, or if they have tried it and it hasn't worked, the next step is short term ( $\leq 4$ weeks) pharmacotherapy treatment with fast-acting benzodiazepines (BZD; e.g. triazolam), benzodiazepine receptor agonists (BZRA; e.g., zolpidem), and certain sedative antidepressants (e.g., trazodone) (Riemann et al., 2017). Guidelines do not recommend phytopharmaceuticals (e.g., valerian), complementary or alternative treatments (e.g., homeopathy) (Riemann et al., 2017). When patients have insomnia and depression, the American Academy of Sleep Medicine (AASM) recommends treating insomnia as stated above and separately prescribing antidepressants or psychotherapy for separately (Schutte-Rodin, Broch, Buysse, Dorsey, \& Sateia, 2008).

Recent studies revealed that PCPs commonly treat insomnia with sleep hygiene alone, pharmacotherapy alone, or both (Bjorvatn, Meland, Flo, \& Mildestvedt, 2017; Conroy \& Ebben, 2015; Everitt et al., 2014; Sivertsen et al., 2010). Sleep hygiene is often the first treatment (Bjorvatn, Fiske, \& Pallesen, 2011; Everitt et al., 2014; Sivertsen et al., 2010) though it is less effective than CBT-I 
1 prescribe medication (Everitt et al., 2014), most commonly benzodiazepines, antidepressants

2 (Bjorvatn et al., 2017; Everitt et al., 2014; Maire et al., 2020; Marschall et al., 2017; Sivertsen et al.,

3 2010), and phytopharmaceuticals (Everitt et al., 2014; Lai, Tan, \& Lai, 2011; Sanchez-Ortuno,

4 Belanger, Ivers, LeBlanc, \& Morin, 2009).

5 The gap between insomnia treatment guidelines and PCPs practice in Europe is troubling and

6 we aimed to investigate whether the trend was similar in Switzerland. We hypothesized that Swiss

7 PCPs would often treat chronic insomnia with medication, that most know little about CBT-I, and that

8 they rarely know specialists who provide CBT-I. We thus created a survey featuring two case

9 vignettes (chronic insomnia and chronic insomnia with comorbid depression) to explore PCPs' usual

10 treatment approach and included questions about their knowledge of CBT-I and CBT-I providers. 


\section{Methods}

\section{Participants}

3 We invited all Swiss PCPs working as medical student preceptors for the Institute of Primary Care at the University of Bern (BIHAM) and all PCPs who report for the Sentinella practice-based research network (PBRN) ${ }^{1}$ led by the Federal Office of Public Health (BAG) to participate in a survey. PCPs who belonged to both groups were asked to complete only one survey. The participation in the survey was voluntary and the data was collected anonymously. PCPs were excluded from the survey if they worked only as PCP pediatricians.

\section{Survey development and structure}

Our survey comprised three parts. Part 1 collected demographic data about the PCPs: sex; age; years of work experience (divided into age groups, e.g., 5-10 years, 11-15 years); practice site (urban, intermediate, rural); and, medical qualifications related to sleep medicine (pneumology, psychiatry, neurology, psychosomatic and psychosocial medicine). Part 2 consisted of two case vignettes, one presenting a case of chronic insomnia without any comorbidity, the other a case of co-occurring chronic insomnia and major depression. PCPs were asked to decide on the treatment approach they would take in each case. Options were divided in pharmacological, non-pharmacological, and complementary-alternative treatment. Each answer block contained a selection of common treatment approaches (see Supplementary Table 1) and a free text field to capture methods not listed. PCPs could choose more than one answer. Part 3 requested PCPs to rate on 5-point Likert scales their knowledge of CBT-I, their interest in learning more about pharmacological and non-pharmacological treatments for chronic insomnia, and asked to indicate if they knew of a nearby professional who offered CBT-I. PCPs were also asked how often they feel their chronic insomnia patients expect them to prescribe a hypnotic. Several professionals in sleep medicine, psychiatry and general medicine reviewed the survey before we distributed it. We developed the online survey with Ilias, a content development tool for educators provided by the University of Bern, Switzerland. We provided the survey in either German or French and piloted it with five PCPs, whose feedback we used to improve it. Anonymized surveys fall outside the Swiss Human Research Act, so our study did not need to be approved by an ethics committee.

\section{Procedures}

The PCPs who worked as medical student preceptors received the link to the survey in an electronic newsletter, via email. We sent two email reminders to non-responders in this group and mailed them a paper-pencil version of the survey if they failed to respond. The PCPs involved in the Sentinella project took part in a parallel data collection on chronic insomnia prevalence and were mailed the paper-pencil version by post. Sentinella non-responders were mailed one written reminder. Data was collected from May to September 2018.

\footnotetext{
${ }^{1}$ A network initially designed to collect data on communicable diseases such as influenza
} 


\section{Statistical analysis}

2 To describe baseline characteristics, we reported continuous variable data in means and standard

3 errors (SE), and categorical variable data in percentages. To assess the treatment PCPs initiated in the

4 case vignettes, we calculated the proportions of PCPs who chose each treatment option. To compare

5 the treatment chosen in the two case vignettes, we used Fisher's exact test. After Bonferroni-

6 correction for multiple comparison, we considered a p-value of 0.0024 to be statistically significant.

7 To investigate the association between PCPs demographics and knowledge about CBT-I, we fitted

8 multivariate logistic regression models with CBT-I knowledge and CBT-I provider knowledge as

9 outcomes, and PCPs sex, age, practice site, years of experience, and further qualification as covariates.

10 To investigate the association between PCPs characteristics and their treatment approach in the case

11 vignettes, we fitted a multivariate logistic regression model with treatment as outcome and PCPs sex,

12 age, practice site, years of experience, further qualification, CBT-I knowledge, and CBT-I provider

13 knowledge as covariates. A p-value of $<0.05$ was considered statistically significant. We analyzed

14 available data and did not use statistical procedures to impute missing data because there were few

15 missing values in the final data set (see Supplementary Table 2). When PCPs did not answer the

16 treatment question at all, we marked the answer as missing. STATA 15.1 was used for all analyses

17 (StataCorp, College Station, TX, USA). 


\section{$1 \quad$ Results}

\section{Demographics}

3 A total of 820 PCPs were invited: 693 worked as medical student preceptors, 132 were PCPs in the

4 Sentinella research network. Five PCPs belonged to both groups. Survey response rate was 48\%

$5(n=395 / 820)$ for all groups: $70 \%(n=93 / 132)$ in the Sentinella group and $44 \%(n=302 / 688)$ in the

6 student preceptor group (41\% online, 59\% per post). We excluded 34 surveys; 18 PCPs were only

7 working as pediatricians and 16 PCPs didn't finish the survey. In the end, we analyzed 361 surveys;

$870 \%(n=252 / 358)$ the respondents were men, 90\% $(n=323 / 361)$ were German speaking; mean age was

954 years. Nearly half $(43 \%, n=154 / 360)$ had been practicing over 20 years. Practice locations were

10 evenly distributed across urban, intermediate, and rural. (See Table 1 for a detailed description of

11 participants.)

12

13 Table 1 Demographic and occupational characteristics of respondents.

\begin{tabular}{|c|c|c|}
\hline Characteristics $(\mathrm{N}=361)^{*}$ & $\begin{array}{l}\mathrm{N} \text { or } \\
\text { mean }\end{array}$ & $\%$ or SE \\
\hline \multicolumn{3}{|l|}{ Sex } \\
\hline Female & 106 & $29.4 \%$ \\
\hline Male & 252 & $69.8 \%$ \\
\hline \multicolumn{3}{|l|}{ Mean Age } \\
\hline & 54.3 & 0.5 \\
\hline \multicolumn{3}{|l|}{ Language } \\
\hline German & 323 & $89.5 \%$ \\
\hline French & 38 & $10.5 \%$ \\
\hline \multicolumn{3}{|l|}{ Experience as $\mathrm{PCP}$} \\
\hline$<5$ years & 31 & $8.6 \%$ \\
\hline $5-10$ years & 58 & $16.1 \%$ \\
\hline $11-15$ years & 65 & $18.0 \%$ \\
\hline $16-20$ years & 52 & $14.4 \%$ \\
\hline$>20$ years & 154 & $42.7 \%$ \\
\hline \multicolumn{3}{|l|}{ Practice location } \\
\hline Urban & 119 & $33.0 \%$ \\
\hline Intermediate & 99 & $27.4 \%$ \\
\hline Rural & 143 & $39.6 \%$ \\
\hline \multicolumn{3}{|l|}{ Further qualification } \\
\hline Pneumology & 2 & $0.6 \%$ \\
\hline PPM & 33 & $9.1 \%$ \\
\hline None & 326 & $90.3 \%$ \\
\hline \multicolumn{3}{|c|}{$\begin{array}{l}\text { *Incomplete demographic data in } 4 \text { participants } \\
\text { PCP = primary care physician, PPM: } \\
\text { psychosomatic and psychosocial medicine, SE: } \\
\text { standard error }\end{array}$} \\
\hline
\end{tabular}

\section{Case Vignettes}

16 Almost all PCPs initiated both pharmacological and non-pharmacological treatment in both cases.

17 Figures 1, 2 and 3 provide an overview of the pharmacological, non-pharmacological and

18 complementary-alternative treatments PCPs initiated in the case vignettes. 


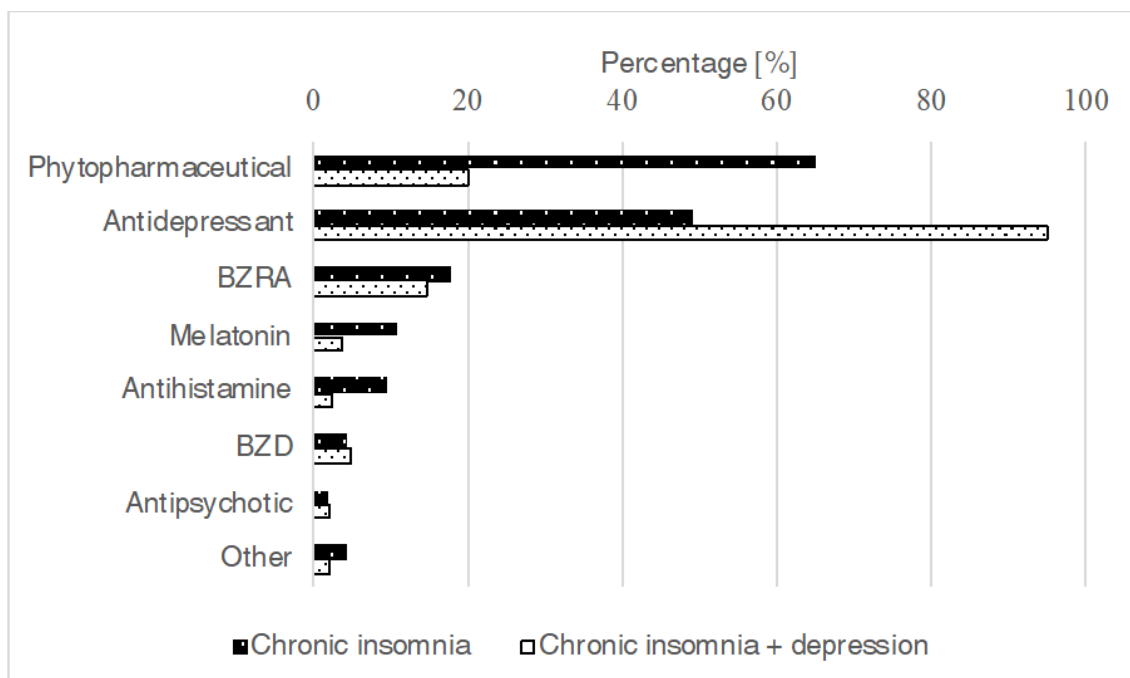

2 Fig. 1 Pharmacological treatment initiated by primary care physicians regarding two cases of 3 insomnia.

$4 \quad \mathrm{BZD}=$ benzodiazepine, $\mathrm{BZRA}=$ benzodiazepine receptor agonist.

5

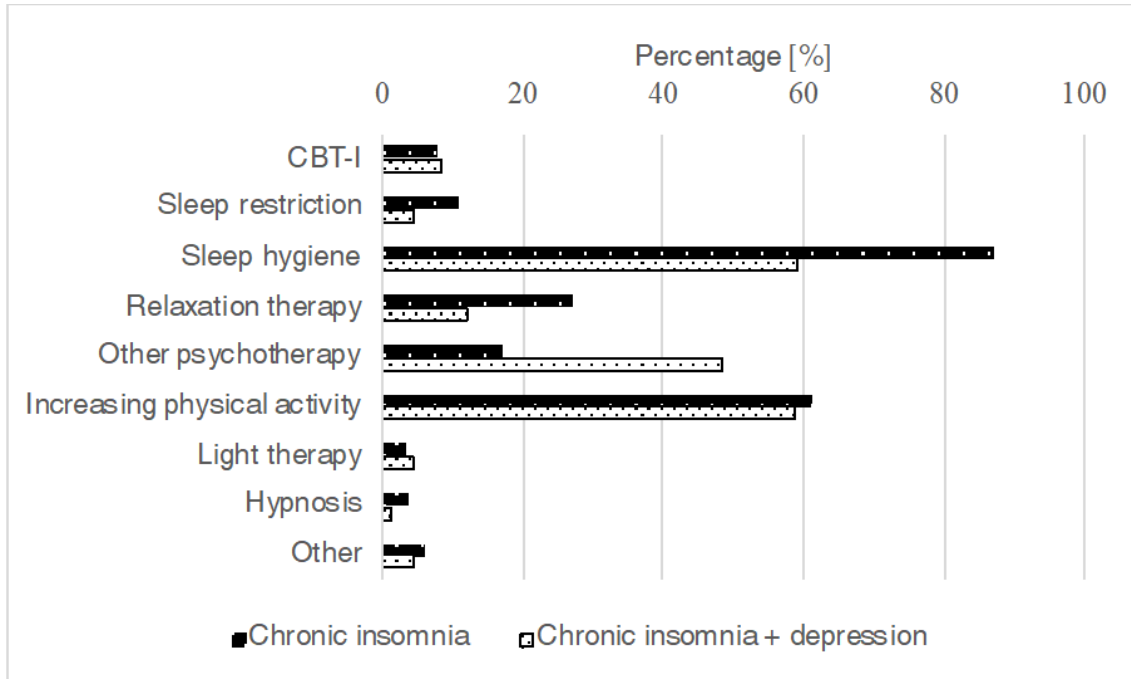

7 Fig. 2 Non-pharmacological treatment initiated by primary care physicians regarding two cases of 8 insomnia.

$9 \quad \mathrm{CBT}-\mathrm{I}=$ cognitive behavioral therapy for insomnia.

10

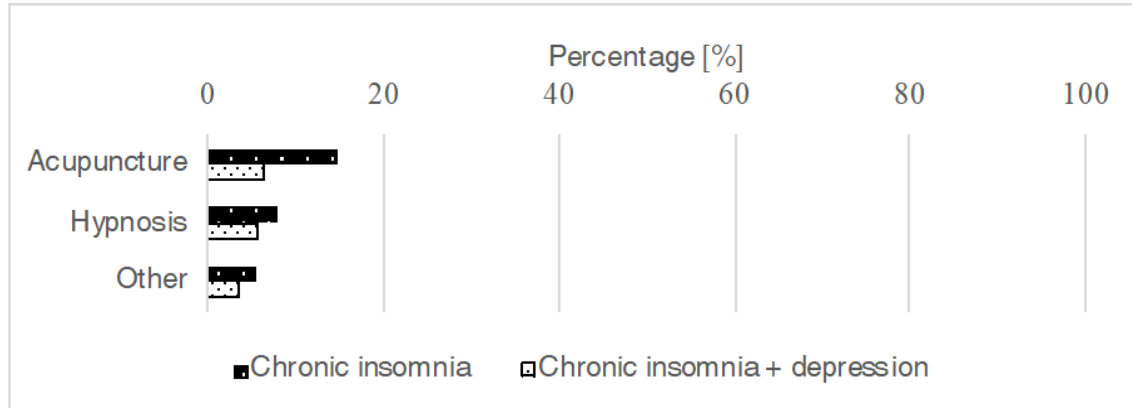

12 Fig. 3 Complementary-alternative treatment initiated by primary care physicians regarding two cases of insomnia. 
2 Two-thirds of all PCPs $(65 \%, n=235 / 361)$ prescribed phytopharmaceuticals and half prescribed antidepressants $(49 \%, n=177 / 361)$, a fifth prescribed BZRA $(18 \%, n=64 / 361)$, and $4 \%(n=15 / 361)$

4 prescribed BZD. The most commonly initiated non-pharmacological treatments were sleep hygiene $(87 \%, n=314 / 361)$ and physical activity advice $(61 \%, n=221 / 361)$. A third of PCPs prescribed relaxation therapy $(27 \%, n=98 / 361)$. Few PCPs prescribed psychotherapy $(17 \%, n=61 / 361)$. Even fewer prescribed sleep restriction $(11 \%, n=39 / 361)$, or CBT-I $(8 \%, n=28 / 361) ; 2 \%(n=9 / 361)$ prescribed all three components of CBT-I (i.e. sleep hygiene, relaxation therapy, sleep restriction), but did not explicitly prescribe CBT-I. Almost a quarter $(23 \%, n=82 / 361)$ of PCPs initiated a complementary-alternative treatment, usually acupuncture $(15 \%, n=53 / 361)$ or homeopathy $(8 \%$, $\mathrm{n}=29 / 361$ ). In multivariate adjusted models, male PCPs were less likely to prescribe phytopharmaceuticals (OR 0.36, $\mathrm{p}<0.001 ; 95 \%$ CI $0.20-0.64$ ), sleep hygiene (OR $0.4, \mathrm{p}=0.045 ; 95 \%$ CI $0.16-0.98$ ), and relaxation therapy (OR $0.42, \mathrm{p}<0.001 ; 95 \%$ CI $0.25-0.72$ ). French-speaking PCPs prescribed significantly fewer antidepressants (OR 0.11, $\mathrm{p}<0.001 ; 95 \%$ CI $0.04-0.32$ ). Case 2: chronic insomnia and depression

16 Most PCPs prescribed antidepressants (95\%, $n=343 / 361)$. About one-fifth prescribed 17 phytopharmaceuticals $(20 \%, n=72 / 361)$ and BZRA $(15 \%, n=53 / 361)$. BZD were prescribed by $5 \%$ 18 ( $n=18 / 361)$ of PCPs. The most commonly initiated non-pharmacological treatments were sleep hygiene $(59 \%, n=213 / 361)$, physical activity advice $(59 \%, n=212 / 361)$ and psychotherapy $(48 \%$, $\mathrm{n}=174 / 361)$. Relaxation therapy was prescribed by $12 \%(\mathrm{n}=43 / 361)$. Few prescribed CBT-I $(9 \%$, $n=31 / 361)$ and sleep restriction (4\%, $n=16 / 360)$. One in 10 PCPs prescribed complementaryalternative treatments $(12 \%, n=43 / 361)$, usually acupuncture $(6 \%, n=23 / 361)$ and homeopathy $(6 \%$, $n=20 / 361)$.

24 French-speaking PCPs (OR 0.18, $\mathrm{p}=0.003 ; 95 \%$ CI 0.06 - 0.56) prescribed significantly less antidepressants and were more likely to give sleep hygiene advice (OR 3.13, p=0.01; 95\% CI 1.31 7.52). 
1 Table 2 Covariates associated with the initiated treatment by primary care physicians in a case of 2 insomnia and a case of insomnia comorbid with depression.*

\begin{tabular}{|c|c|c|c|}
\hline \multicolumn{4}{|l|}{ A) Case 1: Insomnia - Treatment } \\
\hline & OR & $95 \% \mathrm{CI}$ & p-value \\
\hline \multicolumn{4}{|l|}{ Antidepressants } \\
\hline French-speaking PCP & 0.11 & $0.04-0.32$ & $<0.001$ \\
\hline \multicolumn{4}{|l|}{ Phytopharmaceuticals } \\
\hline Male PCP & 0.36 & $0.20-0.64$ & $<0.001$ \\
\hline \multicolumn{4}{|l|}{ Sleep hygiene } \\
\hline Male PCP & 0.4 & $0.16-0.98$ & 0.045 \\
\hline \multicolumn{4}{|l|}{ Relaxation therapy } \\
\hline Male PCP & 0.42 & $0.25-0.72$ & 0.001 \\
\hline \multicolumn{4}{|l|}{ CBT-I } \\
\hline CBT-I knowledge & 2.59 & $1.61-4.15$ & $<0.001$ \\
\hline CBT-I provider knowledge & 6.55 & $2.92-14.67$ & $<0.001$ \\
\hline
\end{tabular}

\begin{tabular}{lccc}
\hline B) Case 2: Insomnia + Depression - Treatment & & & \\
& OR & $95 \% \mathrm{CI}$ & p-value \\
\hline $\begin{array}{l}\text { Antidepressants } \\
\quad \begin{array}{l}\text { French-speaking PCP } \\
\text { Sleep hygiene }\end{array} \\
\quad \text { French-speaking PCP }\end{array}$ & 0.18 & $0.06-0.56$ & 0.003 \\
CBT-I $\quad 3.13$ & $1.31-7.52$ & 0.01 \\
$\quad$ CBT-I knowledge & & & \\
$\quad$ CBT-I provider knowledge & 2.69 & $1.7-4.24$ & $<0.001$ \\
& 6.44 & $2.95-14.05$ & $<0.001$
\end{tabular}

A complete list of treatment options is available online (Supplementary Table 1) $C B T-I=$ cognitive behavioral therapy for insomnia, $P C P=$ primary care physician.

* Covariates used for multivariate adjustment: sex, mean age, language, experience as PCP, practice location, further qualification, knowledge about CBT-I and CBT-I providers. Only treatment options with significantly associated covariates are presented.

\section{Comparing treatment modalities of PCPs between case 1 and 2}

When both insomnia and depression were present, PCPs were significantly more likely to prescribe antidepressants ( $95 \%$ vs. $49 \%$ ) and psychotherapy ( $48 \%$ vs. $17 \%$ ). Phytopharmaceuticals were prescribed three times less in this case ( $20 \%$ vs. $65 \%$; all Fisher's exact $=0.000)$.

CBT-I was provided at the same low rate in both case vignettes (9\% and 8\%); CBT-I components like sleep hygiene ( $59 \%$ vs. $87 \%$; Fisher's exact $<0.0001)$, relaxation therapy ( $12 \%$ vs. $27 \%$; Fisher's exact $<0.0001)$ and sleep restriction ( $4 \%$ vs. $11 \%$; Fisher's exact=0.001), were prescribed less often when both insomnia and depression were present. Half as many PCPs initiated a complementaryalternative treatment ( $12 \%$ vs. $23 \%$; Fisher's exact $<0.0001)$ when both conditions were present. The prescription rate of physical activity advice (59\% and 61\%) and hypnotics (20\% and $22 \%)$ were similar in each case.

\section{Perception of chronic insomnia treatment}

About two-thirds of PCPs answered that they knew nothing $(19 \%, n=68 / 360)$ or very little $(46 \%$, $\mathrm{n}=166 / 360)$ about CBT-I (Figure 4). About a fifth of PCPs $(22 \%, \mathrm{n}=80 / 358)$ did know of a local specialist who provided CBT-I (Figure 5). None of the characteristics we evaluated predicted the level of knowledge PCPs had about either CBT-I or CBT-I providers. We found that about two thirds of PCPs prescribing CBT-I had at least moderate knowledge about CBT-I (case $1: \mathrm{n}=18 / 28$, case 2 : 
$1 \mathrm{n}=22 / 31$ ); roughly $60 \%$ of PCPs who prescribed CBT-I in the case vignettes knew a provider (case 1 :

$2 \mathrm{n}=17 / 28$, case $2: \mathrm{n}=18 / 31)$.

3 Over three-quarters of participants said they had great or very great interest in learning more about

4 pharmacological $(76 \%, n=274 / 360)$ and non-pharmacological $(78 \%, n=281 / 361)$ treatments for

5 chronic insomnia. About half of PCPs $(54 \%, n=195 / 361)$ reported they frequently felt patients with

6 chronic insomnia expected them to prescribe a hypnotic.

7

8

9 Fig. 4 Primary care physicians` self-assessed knowledge about cognitive behavioral therapy for 10

11

12

13

14 insomnia.

Fig. 5 Primary care physicians ${ }^{`}$ knowledge of a specialist in their surroundings offering cognitive behavioral therapy for insomnia.

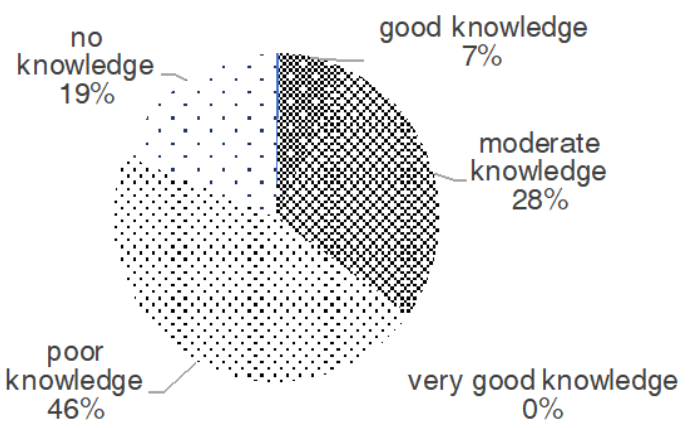




\section{Discussion}

2 PCPs in Switzerland rarely followed evidence-based guidelines when treating chronic insomnia. Instead of CBT-I, they usually prescribed sleep hygiene (87\%), more physical activity (61\%), phytopharmaceuticals (65\%), and antidepressants (49\%) in a clinical vignette presenting a patient with insomnia without depression. A fifth of PCPs prescribed BZRA (18\%) or BZD (4\%). Only 8\% of PCPs initiated CBT-I, the first-line recommended treatment according to guidelines. In presence of a comorbid depression, PCPs were more likely to treat with antidepressants (95\%) and psychotherapy (48\%) and less likely to prescribe phytopharmaceuticals (20\%), sleep hygiene (59\%), or other components of CBT-I. Half of all PCPs reported expectations of patients to prescribe them hypnotics (54\%). Most were unfamiliar with CBT-I (65\%); only a fifth knew a local CBT-I provider (22\%). Most PCPs expressed strong interest in learning more about how to treat chronic insomnia (77\%). These findings confirmed our hypothesis that Swiss PCPs, like those in other European countries, rarely followed the guidelines that recommend CBT-I as a first line treatment for insomnia (Riemann et al., 2017).

Koffel et al. found that, on the system level, PCPs and patients may not have access to CBT-I providers, which are mainly, psychiatrists, clinical psychologists and sleep centers (Koffel, Bramoweth, \& Ulmer, 2018). There are effective online versions of CBT-I, which could reduce this bottleneck if PCPs were aware of them (Krieger et al., 2019; Seyffert et al., 2016). At the clinical level, Koffel et al. identified four barriers to CBT-I utilization: 1) widespread ignorance of CBT-I, which accords with our findings that only $8 \%$ of PCPs prescribed CBT-I; 2) the tendency of PCPs to regard chronic insomnia as a secondary condition that would resolve if the condition they thought primary was treated (Sake, Wong, Bartlett, \& Saini, 2017; Ulmer et al., 2017), which accords with our finding that PCPs tended to treat comorbid insomnia based on depression guidelines (Kupfer, Frank, \& Phillips, 2012); 3) PCPs think patients prefer medication (Cheung et al., 2014), and our findings support this since half of our study PCPs felt pressure to prescribe hypnotics. However, nonpharmacological treatment was prescribed more often than pharmacological treatment; 4) clinicians may lack of motivation, time and resources to manage insomnia (Koffel et al., 2018). Our inquiries did not cover this aspect, but our data suggest that most PCPs are very interested in learning more about treating insomnia, and might even be willing to implement CBT-I themselves.

Sleep hygiene was the most common component of CBT-I prescribed though it is not recommended as a sole treatment for chronic insomnia (Riemann et al., 2017) and may increase drug use (Bjorvatn et al., 2011); 10\% of PCPs prescribed sleep restriction (the most effective single component of CBT-I) (Krieger et al., 2019), and a third prescribed relaxation therapy; $2 \%$ prescribed all three components of CBT-I, but did not explicitly prescribe CBT-I. Common use of CBT-I components indicates that PCPs were willing to treat patients with non-pharmacological options, indicating they may be receptive to prescribing CBT-I. 
Phytopharmaceuticals were the most frequently prescribed medications in our study, though evidence of beneficial effects is lacking and guidelines do not recommend them (Riemann et al., 2017). Young women and well-educated patients are most likely to take phytopharmaceuticals to improve sleep and may prefer them because they are unaware of the lack of efficacy, drug interactions and side effects (Sanchez-Ortuno et al., 2009). Both patients and PCPs may be encouraged to prescribe phytopharmaceuticals because direct-to-consumer advertising suggests they are purely beneficial (Brody \& Light, 2011). In another study, we found a lower rate of phytopharmaceutical and high rate of BZD use in Swiss primary care chronic insomnia patients (Maire et al., 2020). One hypothesis might be that PCPs initially prescribed phytopharmaceuticals and then, because patients do not respond to this ineffective treatment, felt entitled to switch patients to the more effective hypnotics, even though that meant potential long-term treatment and addiction (Schonmann et al., 2018).

The high prescription rate of antidepressants in our study supports data on chronic insomnia patients (Lai et al., 2011; Maire et al., 2020). PCPs may believe antidepressants are the most effective long-term treatment for chronic insomnia (Sivertsen et al., 2010), that they are safer and less addictive than hypnotics (Everitt et al., 2014), or that chronic insomnia is a secondary condition of depression (Sivertsen et al., 2010). They may find antidepressant treatment improves chronic insomnia symptoms when depression is present and conclude it effectively treats chronic insomnia alone (Lai et al., 2011; Sivertsen et al., 2010).

Our PCPs said they would prescribe hypnotics - BZD and BZRA- at a lower rate than PCPs in other countries (Sivertsen et al., 2010). Parallel data collection in Swiss primary care practices revealed that half of chronic insomnia patients in Swiss primary care still use BZD or BZRA for sleep problems (Maire et al., 2020), perhaps because patients are already dependent on them or pressure PCPs to prescribe (Everitt et al., 2014).

In cases of chronic insomnia with depression, PCPs tended to treat depression with antidepressants and psychotherapy rather than CBT-I or its components, suggesting they believe insomnia is a symptom resulting of depression. When PCPs recognize insomnia as an independent condition, patients are less likely to develop chronic insomnia and depression; chronic insomnia doubles a patient's risk of developing depression (Baglioni et al., 2011; Riemann et al., 2020).

The study has four limitations. PCPs self-reported treatment based on fictive case vignettes and not on actual patient data by chart review, for example. PCPs read a short introductory text to the questionnaire. No further instruction on filling out the survey was provided. Second, specifying and categorizing treatment options on the survey form may have biased PCP responses by presenting them with plausible treatment options they might not otherwise have considered; it is also possible our categorizations could have influenced their treatment choice. Third, PCPs who train students or who take part in the Sentinella project may know more about insomnia treatment than the average PCP. PCPs may tend to overestimate their knowledge about CBT-I because it is socially desirable to be 
more informed. As a result, we might have overestimated how much PCPs knew about CBT-I, but this bias would only strengthen our main finding that CBT-I appears vastly under-prescribed and that physicians reported knowing little about it. Fourth, our sample size was too small to use a logistic regression model to test if CBT-I knowledge or CBT-I provider knowledge was associated with CBT-I prescription ( $\mathrm{n}=28$ prescribing CBT-I in case 1 and $n=31$ in case 2 ), so we limited our analyses to descriptive statistics.

The response rate in the Sentinella group (70\%) was substantially higher than in the student preceptor group (44\%). Since the Sentinella PCPs are specifically recruited to participate in data collection and receive a yearly reimbursement for their overall participation, this could explain the much higher response rate in this group. Student preceptor PCPs also seemed to favor the paper-pencil version of the survey over the online version.

PCPs knew little about CBT-I, were not connected to CBT-I providers, often prescribe medication as a first-line treatment, and may still hold the outdated notion that chronic insomnia is a secondary condition of depression. However, non-pharmacological therapies were frequently chosen to treat both insomnia and insomnia comorbid with depression, showing that these therapy options are generally well accepted by PCPs. Since PCPs expressed strong interest in learning more about treatment options for chronic insomnia and may be familiar with some CBT-I components, researchers should identify specific barriers to CBT-I implementation in clinical practice, devise and test interventions that educate PCPs about chronic insomnia treatment, and connect PCPs with specialists who provide CBT-I or familiarize them with online CBT-I (Baglioni et al., 2020).

Our results suggest that informing PCPs about the benefits of CBT-I and connecting them with local CBT-I providers could increase the proportion of Swiss PCPs who prescribe the first-line treatment recommended by guidelines and also reduce the number of patients treated with medication, especially in the presence of depression.

\section{Acknowledgements}

We thank the student preceptor physicians from the University of Bern, the physicians from Sentinella network and the Sentinella administration as well as the Section Notification Systems at the Federal Office of Public Health for their help collecting this data. We thank Sven Streit, Roman Hari, Vivien Bromundt and Corrado Carbazza for their helpful comments on the survey. We thank Reto Auer, Sven Streit, Claudio Cahenzli, Roman Hari and Pascal Baschung for piloting the survey. We thank Eva Gosteli and Nicole Ehrenzeller for their help in survey distribution. 


\section{References}

Association, A. P. (2013). Diagnostic and Statistical Manual of Mental Disorders, DSM-5. .

Baglioni, C., Altena, E., Bjorvatn, B., Blom, K., Bothelius, K., Devoto, A., . . Riemann, D. (2020). The European Academy for Cognitive Behavioural Therapy for Insomnia: An initiative of the European Insomnia Network to promote implementation and dissemination of treatment. $J$ Sleep Res, 29(2), e12967. doi:10.1111/jsr.12967

Baglioni, C., Spiegelhalder, K., Nissen, C., \& Riemann, D. (2011). Clinical implications of the causal relationship between insomnia and depression: how individually tailored treatment of sleeping difficulties could prevent the onset of depression. The EPMA journal, 2(3), 287-293. doi:10.1007/s13167-011-0079-9

Bjorvatn, B., Fiske, E., \& Pallesen, S. (2011). A self-help book is better than sleep hygiene advice for insomnia: a randomized controlled comparative study. Scand J Psychol, 52(6), 580-585. doi:10.1111/j.1467-9450.2011.00902.x

Bjorvatn, B., Meland, E., Flo, E., \& Mildestvedt, T. (2017). High prevalence of insomnia and hypnotic use in patients visiting their general practitioner. Fam Pract, 34(1), 20-24. doi:10.1093/fampra/cmw107

Brody, H., \& Light, D. W. (2011). The inverse benefit law: how drug marketing undermines patient safety and public health. American journal of public health, 101(3), 399-404. doi:10.2105/AJPH.2010.199844

Calem, M., Bisla, J., Begum, A., Dewey, M., Bebbington, P. E., Brugha, T., . . Stewart, R. (2012). Increased prevalence of insomnia and changes in hypnotics use in England over 15 years: analysis of the 1993, 2000, and 2007 National Psychiatric Morbidity Surveys. Sleep, 35(3), 377-384. doi:10.5665/sleep. 1700

Cheung, J. M. Y., Atternas, K., Melchior, M., Marshall, N. S., Fois, R. A., \& Saini, B. (2014). Primary health care practitioner perspectives on the management of insomnia: a pilot study. Australian journal of primary health, 20(1), 103-112. doi:10.1071/PY12021

Conroy, D. A., \& Ebben, M. R. (2015). Referral Practices for Cognitive Behavioral Therapy for Insomnia: A Survey Study. Behav Neurol, 2015, 819402. doi:10.1155/2015/819402

Delini-Stula, A., Bischof, R., \& Holsboer-Trachsler, E. (2007). Sleep behavior of the Swiss population: Prevalence and the daytime consequences of insomnia. Somnologie, 11(3), 193201. doi:10.1007/s11818-007-0297-5

Everitt, H., McDermott, L., Leydon, G., Yules, H., Baldwin, D., \& Little, P. (2014). GPs' management strategies for patients with insomnia: a survey and qualitative interview study. Br J Gen Pract, 64(619), e112-119. doi:10.3399/bjgp14X677176

Koffel, E., Bramoweth, A. D., \& Ulmer, C. S. (2018). Increasing access to and utilization of cognitive behavioral therapy for insomnia (CBT-I): a narrative review. doi:10.1007/s11606-018-4390-1

Krieger, T., Urech, A., Duss, S. B., Blattler, L., Schmitt, W., Gast, H., . . Berger, T. (2019). A randomized controlled trial comparing guided internet-based multi-component treatment and internet-based guided sleep restriction treatment to care as usual in insomnia. Sleep Medicine, 62, 43-52. doi:10.1016/j.sleep.2019.01.045

Kupfer, D. J., Frank, E., \& Phillips, M. L. (2012). Major depressive disorder: new clinical, neurobiological, and treatment perspectives. Lancet, 379(9820), 1045-1055. doi:10.1016/S0140-6736(11)60602-8

Lai, L. L., Tan, M. H., \& Lai, Y. C. (2011). Prevalence and factors associated with off-label antidepressant prescriptions for insomnia. Drug Healthc Patient Saf, 3, 27-36. doi:10.2147/DHPS.S21079

Leger, D., Guilleminault, C., Dreyfus, J. P., Delahaye, C., \& Paillard, M. (2000). Prevalence of insomnia in a survey of 12,778 adults in France. J Sleep Res, 9(1), 35-42. doi:10.1046/j.13652869.2000.00178.x

Li, M., Zhang, X. W., Hou, W. S., \& Tang, Z. Y. (2014). Insomnia and risk of cardiovascular disease: a meta-analysis of cohort studies. Int J Cardiol, 176(3), 1044-1047. doi:10.1016/j.ijcard.2014.07.284

Maire, M., Linder, S., Dvorak, C., Merlo, C., Essig, S., Tal, K., . . Auer, R. (2020). Prevalence and management of chronic insomnia in Swiss primary care: Cross-sectional data from the "Sentinella" practice-based research network. J Sleep Res, e13121. doi:10.1111/jsr.13121 
Marschall, J., Hildebrandt, S., Sydow, H., \& Nolting, H. D. (2017). Gesundheitsreport 2017 (DAK Report). Medhochzwei Verlag.

Miller, C. B., Espie, C. A., Epstein, D. R., Friedman, L., Morin, C. M., Pigeon, W. R., . . Kyle, S. D. (2014). The evidence base of sleep restriction therapy for treating insomnia disorder. Sleep Med Rev, 18(5), 415-424. doi:10.1016/j.smrv.2014.01.006

Novak, M., Mucsi, I., Shapiro, C. M., Rethelyi, J., \& Kopp, M. S. (2004). Increased utilization of health services by insomniacs--an epidemiological perspective. Journal of psychosomatic research, 56(5), 527-536. doi:10.1016/j.jpsychores.2004.02.007

Pigeon, W. R., Funderburk, J., Bishop, T. M., \& Crean, H. F. (2017). Brief cognitive behavioral therapy for insomnia delivered to depressed veterans receiving primary care services: A pilot study. Journal of affective disorders, 217, 105-111. doi:10.1016/j.jad.2017.04.003

Riemann, D., Baglioni, C., Bassetti, C., Bjorvatn, B., Dolenc Groselj, L., Ellis, J. G., . . . Spiegelhalder, K. (2017). European guideline for the diagnosis and treatment of insomnia. Journal of Sleep Research, 26(6), 675-700. doi:10.1111/jsr.12594

Riemann, D., Krone, L. B., Wulff, K., \& Nissen, C. (2020). Sleep, insomnia, and depression. Neuropsychopharmacology : official publication of the American College of Neuropsychopharmacology. doi:10.1038/s41386-019-0411-y

Riemann, D., \& Perlis, M. L. (2009). The treatments of chronic insomnia: a review of benzodiazepine receptor agonists and psychological and behavioral therapies. Sleep Medicine Reviews, 13(3), 205-214. doi:10.1016/j.smrv.2008.06.001

Sake, F. T., Wong, K., Bartlett, D. J., \& Saini, B. (2017). Insomnia Management in the Australian Primary Care Setting. Behav Sleep Med, 17(1), 19-30. doi:10.1080/15402002.2016.1266491

Sanchez-Ortuno, M. M., Belanger, L., Ivers, H., LeBlanc, M., \& Morin, C. M. (2009). The use of natural products for sleep: A common practice? Sleep Med, 10(9), 982-987. doi:10.1016/j.sleep.2008.10.009

Schonmann, Y., Goren, O., Bareket, R., Comaneshter, D., Cohen, A. D., \& Vinker, S. (2018). Chronic hypnotic use at 10 years-does the brand matter? Eur J Clin Pharmacol, 74(12), 1623-1631. doi:10.1007/s00228-018-2531-4

Schutte-Rodin, S., Broch, L., Buysse, D., Dorsey, C., \& Sateia, M. (2008). Clinical guideline for the evaluation and management of chronic insomnia in adults. Journal of clinical sleep medicine : JCSM : official publication of the American Academy of Sleep Medicine, 4(5), 487-504. Retrieved from $<$ Go to ISI $>$ ://MEDLINE: 18853708

https://www.ncbi.nlm.nih.gov/pmc/articles/PMC2576317/pdf/jcsm.4.5.487.pdf

Seyffert, M., Lagisetty, P., Landgraf, J., Chopra, V., Pfeiffer, P. N., Conte, M. L., \& Rogers, M. A. M. (2016). Internet-Delivered Cognitive Behavioral Therapy to Treat Insomnia: A Systematic Review and Meta-Analysis. PLoS One, 11(2), e0149139. doi:10.1371/journal.pone.0149139

Sivertsen, B., Nordhus, I. H., Bjorvatn, B., \& Pallesen, S. (2010). Sleep problems in general practice: a national survey of assessment and treatment routines of general practitioners in Norway. $J$ Sleep Res, 19(1 Pt 1), 36-41. doi:10.1111/j.1365-2869.2009.00769.x

Ulmer, C. S., Bosworth, H. B., Beckham, J. C., Germain, A., Jeffreys, A. S., Edelman, D., . . Voils, C. I. (2017). Veterans Affairs Primary Care Provider Perceptions of Insomnia Treatment. $J$ Clin Sleep Med, 13(8), 991-999. doi:10.5664/jcsm.6702 
1 Supplementary Table 1 Treatment options presented to the primary care physicians.

\begin{tabular}{|l|l|l|}
\hline Pharmacological & Non-pharmacological & Complementary-alternative \\
\hline Phytopharmaceutical & CBT-I & Acupuncture \\
\hline Antidepressant & Sleep restriction & Homeopathy \\
\hline Benzodiazepine receptor agonist & Sleep hygiene & Other (free text) \\
\hline Melatonin & Relaxation therapy & \\
\hline Antihistamine & Other psychotherapy & \\
\hline Benzodiazepine & Increasing physical activity & \\
\hline Antipsychotic & Light therapy & \\
\hline Other (free text) & Hypnosis & \\
\hline & Other (free text) & \\
\hline
\end{tabular}

2

3 Supplementary Table 2 Number of missing values for each variable with missing data.

\begin{tabular}{l|c} 
Variable & Missing values \\
\hline Age & 4 \\
\hline Sex & 3 \\
\hline Experience as a PCP & 1 \\
\hline Knowledge about CBT-I & 1 \\
\hline CBT-I provider knowledge & 3 \\
\hline $\begin{array}{l}\text { Interest in learning more about } \\
\text { pharmacological treatment of } \\
\text { chronic insomnia }\end{array}$ & 1
\end{tabular}

4 